\title{
Research on the Status of Automatic Driving and the Key Technology of Vehicle Network
}

\author{
Yunhua Gao \\ Jiangsu Vocational Institute of Commerce, Nanjing, China
}

Keywords: Automatic driving; Vehicle network technology; DSRC; LTE-V2X;

\begin{abstract}
Automatic driving has gradually come into view. Vehicle network technology extends the vision of automatic driving and is more secure. V2X is the key link of the Internet of vehicles technology and the important means of realizing the intelligent transportation system. V2X obtains the road condition information in real time through the information interaction between vehicles and vehicles, vehicles and road facilities, vehicles and pedestrians, vehicles and the mobile Internet, and then converges and analyzes the information of the external environment to the vehicle terminal, providing intelligent decision-making basis for the vehicle driving, improving traffic management efficiency, and reducing the probability of safety accidents, so as to achieve automatic driving.
\end{abstract}

\section{Introduction}

Now the research of automatic driving car be said to have reached the point of extreme suffering. With the increasing investment of people from all walks of life, the results of automatic driving have constantly appeared before people's eyes. Why do we need it? What's the use of it? As we know, over 63000 road traffic deaths in China in 2017. Over 90\% of accidents caused by human errors. In addition, traffic jams costs Beijing alone $\$ 11.3 \mathrm{~B}$ a year, including time, fuel and pollution. As the number of vehicles increases, parking related issues causes $30 \%$ of the congestion in China. Also there is 35\% of China's population over 60 years old by 2050(WHO), which will cause significant shortages in labor force. Now automatic driving car will continuously monitor the road conditions and reduce accident. When the owner's parking capacity is insufficient, the automatic parking function will bring a very pleasant driving experience. As the automatic driving cars can accurately predict braking time and adjust driving speed, it correspondingly reduce congestion and improve quality of time. This paper aims to comprehensively analyze the current development states and future trend of automatic driving technologies and products, which provides a reference for the R\&D and promotion of autonomous vehicles.

\section{Automatic Driving Architecture}

\subsection{Automatic Driving Grade.}

SAE (American Association of Automobile Engineers) and NHTSA (United States Highway Safety Service) have graded automatic driving technology. SAE divides automatic driving technology into 0 to 5 in six levels. NHTSA divides it into 5 levels of 0 to 4 [1]. Take SAE as an example,

L0: Complete human driving.

L1: Auxiliary driving which increases the ADAS function of alert, including lane departure warning (LDW), pre-collision warning (FCW) and blind spot detection (BSD).

L2: Partial automatic driving which has the ADAS capabilities of the daring auxiliary class, including adaptive cruise (ACC), emergency automatic brake (AEB), lane keeping auxiliary (LKA), etc.

L3: Comprehensive auxiliary functions, including automatic acceleration, automatic braking and automatic turning lights. 
L4: Highly automatic driving, without any human driver, can no steering wheel, throttle, brake pedal, but limited area (such as the park, scenic area), or limited environmental conditions.

L5: fully automatic driving is a truly unmanned phase, with no driver's position and no cognitive intervention in or out of the car.

\subsection{Automatic Driving Hierarchy.}

Automatic driving is divided into three layers: the perception layer, the decision-making layer and the executive layer 。

The perception layer is used to recognize and recognize the surrounding environment of vehicles. Automatic driving car uses a variety of sensors, including cameras, millimeter wave radar, laser radar, ultrasonic radar, infrared night vision, and GPS for positioning and navigation. Another kind of technology is not active detection element, but it is a collaborative global data assistant, which can extend the environment perception ability of intelligent vehicle, including high precision map and V2X vehicle network technology. Each type of perception technology has its own advantages and disadvantages, and they complement each other, and eventually it is a very high security requirement for the smart car to reach the driving scene.

Decision-making layer is the part of artificial intelligence that really works, just like human pilots. The decision-making layer is divided into two steps, the first step cognitive understanding, according to the information collected by the perceptual layer, the precise positioning of the vehicle itself, and the accurate understanding of the environment around the vehicle. The second step of the decision planning, including the accurate prediction of the next possible situation, the judgment and planning of the next step, and the choice of a reasonable path to achieve the goal.

The executive layer is the core of automatic driving, and the development from mechanization to electronic control is the trend. The implementation mechanism mainly includes three major systems: driving, braking, and turning. The development from mechanization to electronic control is the trend. This is mainly due to the fact that the delay time of the electronic control is shorter than that of the mechanical system, and it is closer to the linear control. This avoids the difficulty caused by the nonlinear output characteristics of the mechanical system to perform the instructions accurately. That is why it is easier for electric cars to develop automatic driving. True automatic driving must integrate the decision control information with the vehicle's underlying control system in depth, complete the electronic control of the execution mechanism through the wire control technology, and achieve electronic braking, electronic driving, and electronic steering.

\section{Overview of the Development of Car Networking at Home and Abroad}

Google's self-driving cars rely mainly on radar, sensors and camera video recognition to obtain the status of the surrounding cars. And these only allow the car to see the car and pedestrians in sight, which is not enough for automatic driving. Car networking technology in avoiding traffic accidents, improving road safety and alleviating congestion, improving traffic efficiency, reducing energy consumption and environmental pollution significant results have been achieved in other areas.

The true meaning of car networking is not just limited to cars + nets or cars + cars. It contains the definition of at least four dimensions: cars to cars, cars to infrastructure, cars to pedestrians, cars to the Internet [2]. In other words, the car is a local area network. The car and the car form an inter-vehicle network. Based on a unified agreement, data interchange between people, cars, roads, and clouds is realized, and eventually smart transportation, smart cars, smart driving and other functions are realized.

In future automatic driving applications, V2X communication technology is real one of the most important technologies for present environmental perception, with conventional vehicle-borne lasers radar, millimeter-wave radar, camera, ultrasound, etc.. Knowing that the advantages of equipment complement each other to provide radar for self-driving vehicles Oversight and complex environmental perception. V2X communications through and from surrounding vehicles, roads, infrastructure,

From the perspective of time and space, the vehicle's perception of traffic and the environment has 
been expanded, and it can be informed in advance of the surrounding vehicle operation information, traffic control information, congestion prediction information, visual blind area and other surrounding environmental information. V2X can be used to enhance environmental awareness, reduce the cost of in-vehicle sensors, and enable more car traffic breath integration decision [3].

\section{Key Technology and Architecture of V2X}

At present, the world's mainstream technology for V2X communications includes dedicated short-range communication (DSRC) technology [4] And LTE-V2X technology.

DSRC is based on IEEE 802.11p and IEEE 802.11a protocol group. The wireless communication technology can provide high-speed data transmission services between vehicles, road vehicles, and intelligent transportation systems in a high-speed moving environment. It has the characteristics of high data transmission rate and short transmission delay. The DSRC operates in the $5 \mathrm{GHz}$ frequency band and supports V2V communications within $300 \mathrm{M}$. The delay is less than $50 \mathrm{MS}$. At $10 \mathrm{MHz}$ system operating bandwidth, the data transmission rate can reach $3 \sim 27 \mathrm{Mbit} / \mathrm{s}$, and the complexity of implementation is low. It can be seen that DSRC has superior performance compared to Wi-Fi.

LTE-V2X is based on 4G technology to realize car and car communication, with LTE cellular network as the V2X based proprietary protocol of the network, including two working modes of LTE-V-Cell and LTE-V-Direct.

With the help of existing cellular networks, LTE-V-Cell can support large bandwidth, large coverage communication and meet the needs of telematics applications. LTE-V-Direct can be independent of cellular network to achieve low delay and high reliability direct communication between vehicle and surrounding environment nodes, and meet the traffic safety requirements.

LTE-V-Cell key indicators: the maximum transmission bandwidth can be extended to $100 \mathrm{MHz}$, the peak rate uplink $500 \mathrm{Mbps}$, downlink $1 \mathrm{Gbps}$, time delay user surface delay less than $10 \mathrm{~ms}$, control surface delay less than $50 \mathrm{~ms}$, support vehicle speed $500 \mathrm{~km} / \mathrm{h}$, and the coverage range is similar to LTE range.

LTE-V-Direct has not yet detailed technical specifications. It is reported that LTE-D has the ability to search for thousands of devices and services within 500 meters, so that more than two of the closest LTE-D devices can communicate in the net [5].

\section{The Significance of $\mathbf{5 G}$ to Car Networking and automatic driving}

If the LTE-V2X based on 4G/4 .5 G is not yet able to carry the burden, then the commercial use of $5 G$ provides LTE-V2X with more powerful performance and more possibilities. Unlike 4G, which focuses mainly on interpersonal communication, $5 \mathrm{G}$ forms an end-to-end ecosystem. It enhances mobile bandwidth with a peak rate of up to $20 \mathrm{~Gb} / \mathrm{s}$, supports lower latency $(\leq 10 \mathrm{~ms})$, higher reliability (> $99.99 \%$ ) and greater bandwidth (1 million terminals per square kilometer). These data all mean higher security. After all, millisecond delay has a very different meaning for the discovery and handling of an accident. According to the test results of Huawei and other manufacturers, the 5G-based LTE-V2X is superior to the DSRC in terms of coverage distance and network latency, but the 5G-V2X wants to be commercial and needs to be tested and verified for a longer period of time.

\section{Conclusion}

Although key technologies of automatic driving have achieved great progress, obstacles still exist in the way of realizing commercialization for full automatic driving. At present, the main difficulty is how to reduce the cost, and in real life, the road conditions are varied, very complex, automatic driving, a little careless, will cause casualties, although the traffic regulations are becoming more and more perfect, but there are many people or bicycles that do not obey the traffic rules. Driving automatic driving, but there are many people in the front crossing the traffic lights crossing the road, it may be difficult to make the right judgment. 


\section{References}

[1] Liu Xiaoping. Analysis and Research on the characteristics of fully automatic driverless vehicle [J]. Internal combustion engine Accessories, 2018 (04).

[2] Sun S, Hu J, Peng Y, et al. Support for vehicle-to-everything services based on LTE [J]. IEEE Wireless Communications, 2016, 23(3):4-8.

[3] Bao Haishen. Analysis of next generation vehicle networking V2X technology [J]. Information technology and information technology.2017 (9): 111-114.

[4] SAE J2735, Dedicated Short Range Communications (DSRC) Message Set Dictionary

[5] Chen S, Hu J, Shi Y, etal . LTE-V: ATD_LTE based V2X Solution for Future Vehicular Network [J]. IEEE Internet of Things Journal, 2016. 